\title{
Toward Chemical Signature Analysis of Forensic Evidences
}

\section{Harvey JM Hou*}

Department of Physical Sciences, Alabama State University, Alabama 36104, USA

Forensic evidence analysis is vital in crime investigation and law enforcement. After the forensic evidences are collected in crime scenes and analyzed in forensic laboratory using a variety of experimental techniques, the findings and interpretation of the analytical results are used to solve a crime or dispute in a courtroom. The forensic evidences include forensic drugs, body fluids, explosives, inks, paintings, and biological agents. Most of forensic evidences are complex and contains impurities, such as cutting agents, thinners, adulterants, by-products, and intermediates $[1,2]$. In particular, the crime scenes may include a variety of environment and condition, which may affect the forensic samples and produce impurities. The impurities of the forensic evidences can be used in chemical signature analysis as the chemical fingerprinting or profiling, which provide insightful and specific information in crime investigation.

Forensic signature analysis involves analyzing the samples' composition beyond simple identification and quantitation of forensic evidences. The signature analytical data are able to categorize the forensic samples into similar groups and to offer investigative information of a common origin. In addition, the data may be used for the elucidation of the synthetic pathway or extraction method of evidences, identification of diluents, adulterants, and impurities, and identification of the evidences' geographic origin for plants-derives [1].

Signature analysis of forensic samples includes physical examination and organic and inorganic analysis. The examination deals with color, shape, appearance, and other microscopic characterization of the sample. Chemical analysis of major and minor constituents reveals the information about the samples and the chemical methods used to process and preparation of samples. The information can be related to the environmental conditions prevailing the time and location.

Each stage of the processing and environmental conditions of storage of samples introduces impurities into a patch. The use of impure reagents, dirty glassware, trace metals, residual solvents, and other contaminants are presented in the samples of forensic evidences. Adulterants and diluents added to the batch can provide information regarding batches and groups. These materials tend to be highly variable and may provide unique information for generating link in forensic case investigation $[3,4]$. For instance, cocaine impurities may provide unique and specific information for the origin and chain of transportation $[3,5]$. The gas chromatography-mass spectrometry (GCMS) and liquid chromatography-mass spectrometry (LCMS) are widely used methodologies in identification and quantification of cocaine and its metabolites in forensic samples.

To gain the fundamental knowledge of chemical signature of forensic samples, we are passionate about the response mechanisms of forensic samples to environment using a variety of bioanalytical and biophysical methodologies. We investigated the responses of methamphetamine, phenobarbital, cocaine, marijuana, and diazepam to the UV irradiations and elevated temperatures using bioanalytical methologies [4]. Methamphetamine, phenobarbital, cocaine, marijuana, and diazepam have a high potential for abuse and are among the top list in illicit drug cases in the United States. We observed that methamphetamine, cocaine, and phenobarbital caused the increase of an absorption peak at $960 \mathrm{~nm}$ in aqueous solutions (including acidic, neutral, and basic conditions) with different kinetics upon UV treatment [6]. The lifetimes of the $960-\mathrm{nm}$ peak appearance were between 23.1 to 42.6 min depending on the drugs. Similarly, the elevated temperatures induced the increase of the $960-\mathrm{nm}$ peak in the case of marijuana. The absorption peak at $960 \mathrm{~nm}$ upon UV treatment, may be due to (a) structural rearrangement or isomerization of methamphetamine, (b) a novel unknown compound from the drugs, (c) formation of novel chemical structure between drug and water.

The elevated temperature $\left(100^{\circ} \mathrm{C}\right)$ caused cocaine to have three new absorption peaks at 720,820 , and $960 \mathrm{~nm}$, indicating the thermal sensitivity of cocaine [6]. The GCMS data revealed a GC peak at 7.93 min, which is identified to be cocaine by its mass peaks at $\mathrm{m} / \mathrm{z} 82.09$, 182.13, and 303.20. Upon UV treatment one GC peaks at the retention time of $8.80 \mathrm{~min}$ was observed. We also noticed the consequent GC pattern change of $\sim 10 \mathrm{GC}$ peaks in the range of 2.0-7.5 min. It showed that cocaine appears photo and thermal susceptible. The GCMS analysis revealed a photo degradation product at $8.80 \mathrm{~min}$. The GC pattern in 20-7.5 min was complex and vulnerable to the experimental conditions and may be the "fingerprint signature of cocaine."

Marijuana, also referred to as cannabis, is a major drug of abuse in the world's organized crime groups/gangs, street marketing, and the most analyzed controlled substance within the forensic science drug chemistry sector. We examined the marijuana samples under temperature manipulation, ranging from $50^{\circ} \mathrm{C}$ to $80^{\circ} \mathrm{C}$ conditions [7] The UV-vis spectroscopic data revealed that the elevated temperature causes four novel absorption peaks at $415 \mathrm{~nm}, 610 \mathrm{~nm}, 665 \mathrm{~nm}$, and $960 \mathrm{~nm}$, suggesting a chemical structure change of Marijuana. The thermal induced structural changes of marijuana seemed to follow the first order kinetics. The activation energy of the reaction was determined. The structural change observed is likely the chemical bond rearrangement or isomerization in Marijuana. Further experiments are conducted to compare this data to those obtained by GCMS. Further research is being conducted to compare this data analysis to the data obtained by GC-MS, to denote such changes.

Flunitrazepam is one type of benzodiazepines, which are controlled substances and belongs to date-rape drugs [8-10]. Flunitrazepam is used to relieve anxiety, insomnia, muscle spasms and epileptic seizures. Although it is essential in treating medical conditions, flunitrazepam

${ }^{*}$ Corresponding author: Harvey JM Hou, Department of Physical Sciences, Alabama State University, 915 South Jackson Street, Montgomery, Alabama, USA, Tel: 1-334-229-5121; E-mail: hhou@alasu.edu

Received November 19, 2013; Accepted November 22, 2013; Published November 25, 2013

Citation: Hou HJM (2013) Toward Chemical Signature Analysis of Forensic Evidences. J Forensic Res 4: e113. doi:10.4172/2157-7145.1000e113

Copyright: @ 2013 Hou HJM. This is an open-access article distributed under the terms of the Creative Commons Attribution License, which permits unrestricted use, distribution, and reproduction in any medium, provided the original author and source are credited. 
is one of the misused drugs on the market. Flunitrazepam has several major side effects like drowsiness, dizziness, slurred speech, breathing problems, and rapid heartbeat.

A sensitive a rapid method using gas chromatography with mass spectrometry was developed for simultaneous determination of the benzodiazepines in blood [11]. Liquid chromatography with mass spectrometry has been used in simultaneous analysis of benzodiazepines and metabolites in hair samples [12,13]. We investigated the response of forensic drug flunitrazepam to the UV irradiation by gas chromatography-mass spectrometry [14]. The substantial change in GC pattern involving multiple GC peaks was found, indicating the complex reaction of degradation in flunitrazepam upon UV irradiation. GCMS analysis identified one of the GC components to be amino-flunitrazepam. We also observed the thermal-induced product of flunitrazem to be open-ring component, 4-[2-fluorophenyl-4-[2methylamino-5-nitrophenyl]-3-aza-3-butenoid acid, by GCMS.

The environment in crime scene might involve diverse conditions and may interrupt the detection and identification of human DNA in forensic samples. It is expected that the DNA will be degraded due the exposure of cleaning agents containing strong bases. The DNA profiling of human hair was not observed in the presence of sodium hydroxide, suggesting the $\mathrm{pH}$ of the STR DNA system is critical [15]. The mixture of DNA components including multiple human DNA sources and bacterial DNA contamination are often encountered in forensic cases. We found that the E. coli DNA affects the shape and intensity of DNA profiling [15]. The sensitivity of DNA profiling may be lower than normal in the presence of bacteria. For example, the vWA loci may be greatly sensitive to bacterial DNA contamination.

Gunshot residue (GSR) is particles produced during the discharge of a firearm and composed of unburned and partially burnt propellant powder, particles from the ammunition primer, smoke, grease, lubricants, and metals from the cartridges as well as the weapon itself. Gunpowder or black powder is classified to smokeless power, including single-based, double-based and triple-based powder, and old black power, which consists of charcoal, potassium, and nitrate. Nitrocellulose is the component that is found in single-based smokeless powder. Nitroglycerin, along with the first component, make up double-based smokeless powder. The last component is called nitrogaunidine. All three components are in triple-based smokeless powder. Triple based is rarely used.

A variety of analytical techniques have been used in GSR detection in crime scene [16], including chemical color test, atomic absorption spectroscopy, inductively coupled plasma, scanning electron microscopy-energy dispersion spectroscopy, gas chromatography, high performance liquid chromatography, capillary electrophoresis, and mass spectrometry. The results showed that the FTIR spectrum of the old black powder, which showed five IR peaks associated with vibration modes of the inorganic nitrate and inorganic carbon [6]. It was dramatically different from that of the smokeless powders, which showed more than ten IR peaks of aliphatic phosphate, aliphatic primary amide, and aliphatic hydrocarbon. The single based smokeless powders showed a strong absorption peak at $1532 \mathrm{~cm}^{-1}$, which was weak or absent in double-based and triple-based samples. The FTIR spectra of the GSR sample showed no changes at $\mathrm{pH} 1.0, \mathrm{pH} 7.0$, and $\mathrm{pH} 13.0$ for two hours, indicating the GSR samples seems stable in environment.

As an emerging field and a national need, microbial forensics has been a significantly expended field in terms of detection and identification of the source of microorganisms in the past ten years $[17,18]$. The detailed characterization of these bacteria, viruses and toxins are especially worthwhile to provide a complete categories and classification of the microorganisms in forensic sciences [19]. Tandem mass spectrometry (MS/MS) is one of the key techniques in the identification of botulinum neurotoxins (BoNTs), which are among the top six species in the list of biological agents. BoNTs are one of the top biological weapons in bioterrorism and biocrimes, may be assessed using the signature analysis and potential building a "BoNT signature" database using mass spectral proteomics [20]. This approach may provide specific and detailed information in detection and identification of BoNTs in microbial forensics and potentially serves a model system to inspire the forensic analysis of other bacteria or viruses to combat bioterrorism and biocrimes.

In summary, the key element in forensic analysis is to match an unknown from crime scene and a known from a suspect and to provide scientific sounding evidence for the lead to solve the legal issue in crime investigation. To this end, the databases, including fingerprint, DNA profiling, firearm information as well as chemical signature profile, are particularly powerful tool. Forensic evidences may generate impurities during their manufacture, storage, and exposure to environment. The effect of diverse environmental conditions, including light, temperature, $\mathrm{pH}$, and bacteria, on the controlled substances is one essential way for the production and generation of their impurities. For instance, the new GC peaks, the GC pattern change, and MS/MS profiles revealed by forensic analysis may serve as "chemical signatures" of evidences. Such information will promote the identification of the origins of the forensic evidences as a "chemical fingerprint" for future reference or construction of "forensic signature" databases in forensic science. The chemical signature analysis of forensic evidences can be used to support forensic community and to develop specific applications in forensic medicine.

\section{References}

1. Bell S (2013) Forensic Chemistry. (2ndedn), Pearson.

2. Stojanovska N, Fu S, Tahtouh M, Kelly T, Beavis A, et al. (2013) A review of impurity profiling and synthetic route of manufacture of methylamphetamine, 3,4-methylenedioxymethylamphetamine, amphetamine, dimethylamphetamine and p-methoxyamphetamine. Forensic Sci Int 224: 8-26.

3. Casale JF, Waggoner RJ (1991) A chromatographic impurity signature profile analysis for cocaine using capillary gas chromatography. J Forensic Sci 36: 1312-1330.

4. Hou HJM (2013a) Response mechanisms of marijuana, cocaine methamphetamine, and flunitrazepam to environment. J Forensic Res 4: 63.

5. Casale JF (2007) Four new illicit cocaine impurities from the oxidation of crude cocaine bases: Formation and characterization of the diasteeomeric 2,3-dihydroxy-3-phenylpropionylecgonine methyl esters from cis- and transcinnamoylcocaine. J Forensic Sci 52: 860-866.

6. Barrow S (2013) Response Mechanisms of Cocaine and Gunshot Residues to Environment. M.S. Thesis. Alabama State University.

7. Phillips L (2013) Structural Changes of Marijuana and Cannabinol Under Diverse Conditions, M.S. Thesis, Alabama State University.

8. LeBeau M, Andollo W, Hearn WL, Baselt R, Cone E, et al. (1999) Recommendations for toxicological investigations of drug-facilitated sexual assaults. J Forensic Sci 44: 227-230.

9. LeBeau M, Mozayani A (2001) Drug-facilitated sexual assaults. Academic Press.

10. Levine B (2010) Principles of Forensic Toxicology (3rdedn). AACC Press.

11. Elian AA (1999) Detection of low levels of flunitrazepam and its metabolites in blood and bloodstains. Forensic Sci Int 101: 107-111. 
12. Kim J, In S, Choi H, Lee S (2013) Illegal use of benzodiazepines and/or zolpidem proved by hair analysis. J Forensic Sci 58: 548-551.

13. Wang KC, Cheng MC, Hsieh CL, Hsu JF, Wu JD, et al. (2013) Determination of nimetazepam and 7-aminonimetazepam in human urine by using liquid chromatography-tandem mass spectrometry. Forensic Sci Int 224: 84-89.

14. Sampson L, Wilson B, Hou HJM (2013b) Gas chromatography-mass spectrometric analysis of forensic drug flunitrazepam upon exposure to UV irradiation. J Forensic Res 4: 193.

15. Sampson L (2013a) Effects of Environmental Factors on Human Hair DNA and Flunitrazepam. M.S. Thesis, Alabama State University.
16. Dalby O, Butler D (2010) Analysis of gunshot residue and associated materials: A review. J Forensic Sci 55: 924-943.

17. Budowle B, Schutzer S, Einseln A, Kelley L, Walsh A, et al. (2003) Public health: Building microbial forensics as a response to bioterrorism. Science 301 : 1852-1853.

18. Budowle B, Schultzer S, Breeze R, Keim P, Morse SA (2010) Microbial Forensics. Academic Press.

19. Hou HJM, Scissum-Gunn K, Wu H, Akujuobi C (2012) Microbial forensics: An emerging field and a national need. Air Water Borne Diseases 1: e107.

20. Hou HJM (2013b) Analysis of botulinum neurotoxin detection by mass spectrometry in forensic samples, J Forensic Res 4: 185. 